\title{
A REVIEW OF 102 CASES OF BEAT CONDITIONS OF THE KNEE
}

\author{
BY
}

\author{
W. B. ROANTREE \\ From the South Eastern Division, National Coal Board
}

(RECEIVED FOR PUBLICATION MAY 9, 1957)

In mining, as in other occupations, entry into the groups qualifying for the higher wage rates is determined by qualification as a skilled man. For the underground worker this means production at, or close to, the coal face, and in coalfields in which the seams are comparatively thin, much of the miner's working time is spent in the performance of hard work while kneeling. With seams about $3 \mathrm{ft}$. in thickness, anything from $25 \%$ to $100 \%$ of the working shift of seven hours or so may be spent on the knees.

The common disorders of the knee which occur in men working in thin seams of coal are very well known to miners under the collective term of " beat knee". Beat knee is a prescribed disease under the National Insurance (Industrial Injuries) Act 1946, and is defined officially as " subcutaneous cellulitis or acute bursitis arising at or about the knee". Although the definition excludes synovial effusions into the knee joint, internal derangements of the joint, osteo-arthritis, and other morbid conditions well represented in miners, the incidence of beat knee is high. The number of cases receiving injury benefit for this condition averaged 10,305 per annum in Great Britain for the three years 1952-54.

The present paper is based on 102 cases of beat knee seen consecutively in a period of two and a half years in one area of the North Eastern (Yorkshire) Division of the National Coal Board. All were seen at colliery medical centres by the writer, and were thus judged independently of any question of their subsequent certification as cases of beat knee under the Industrial Injuries Act.

The material was obtained from seven neighbouring collieries which were well equipped and served by modern medical centres and pit-head baths. Environmental and geological conditions were comparable in the group, and the former may be summarized as follows: the working places were not deep, and were in the region of 1,000 feet below surface; ventilation and environmental working conditions were good, there being neither excessive heat nor water. The coal seams worked were generally thin, so that space was confined and work had to be done on the knees. An estimate of the thickness of the seam in the working places was obtained from 80 men. The seams were $2 \mathrm{ft} .6 \mathrm{in}$. or less in 35 instances, between $2 \mathrm{ft} .9 \mathrm{in}$. and $3 \mathrm{ft} .6$ in. in 38, and chiefly between $3 \mathrm{ft} .9 \mathrm{in}$. and $4 \mathrm{ft}$. in the remaining seven.

\section{Classification}

It was found possible to classify all cases in one of three groups: (1) Inflammatory lesions, 43; (2) pressure lesions, 51 ; (3) other conditions, 8. As pressure and inflammatory lesions differ in aetiology, course, and treatment each will be considered separately.

\section{Inflammatory Lesions}

Inflammatory lesions usually started as infected hair follicles on the anterior or antero-lateral aspect of the knee. Many such follicular lesions run a mild course and heal with no loss of working time if situated in areas which are not subjected to occupational pressure. Miners with early follicular infections continue their work until prevented from doing so by pain or constitutional symptoms. There can be no doubt that the transmission of weight through infected foci exercises an unfavourable effect upon natural attempts at localization, with the subsequent development of follicular abscesses, commonly with a circumscribed zone of cellulitis, usually accompanied by enlarged and tender subinguinal nodes. When localization has failed altogether, a more extensive infection results. Thirteen cases were severe, 18 of moderate severity, and 12 were mild.

Follicular abscesses were present in 33 out of the 43 cases in this group. In 13 there was a single abscess; nine cases had enlarged and tender subinguinal nodes. Ten patients had single abscesses 
TABLE 1

RESULTS OF BACTERIOLOGICAL EXAMINATION

\begin{tabular}{|c|c|c|c|c|c|c|}
\hline \multirow{2}{*}{ Organism } & \multirow{2}{*}{ Coagulase Test } & \multicolumn{4}{|c|}{ Antibiotic Sensitivity in Vitro } & \multirow{2}{*}{ Cases } \\
\hline & & Penicillin & Streptomycin & Chloramphenicol & Aureomycin & \\
\hline \begin{tabular}{cc} 
Staphylococcus & aureus \\
,$"$ & \multicolumn{1}{c}{ albus } \\
$"$, & aureus
\end{tabular} & $\begin{array}{l}+ \\
+ \\
+ \\
-\end{array}$ & $\begin{array}{c}\text { Sensitive } \\
\text { ", } \\
\begin{array}{c}\text { Slightly or very } \\
\text { slightly }\end{array}\end{array}$ & $\begin{array}{c}\text { Sensitive } \\
\text { ", } \\
\text { ", } \\
-\end{array}$ & $\begin{array}{c}\text { Sensitive } \\
\text { ", } \\
\text { " } \\
-\end{array}$ & $\begin{array}{c}\text { Sensitive } \\
\text {," } \\
\text { ", } \\
-\end{array}$ & $\begin{array}{r}11 \\
1 \\
1 \\
4 \\
4\end{array}$ \\
\hline & & & & & & 21 \\
\hline
\end{tabular}

* In one of these the staphylococcus was also sensitive to erythromycin.

** The cultures in one case were sensitive in vitro to erythromycin and chloramphenicol, slightly sensitive to penicillin, and sensitive to streptomycin, aureomycin, and terramycin, with a few resistant colonies.

with limited zones of cellulitis, seven of these being complicated by node enlargement and one by lymphangitis. There were three instances of extensive cellulitis arising from follicular abscesses.

Multiple follicular abscesses, often bilateral, were observed in seven men, two of whom had local cellulitis.

An acute spreading cellulitis without a follicular lesion was seen in 10 cases: six were severe and four subsiding. Constitutional symptoms usually accompanied severe cellulitis, but nodes were involved in one only.

Bacteriology.-Samples were collected by incising small follicular abscesses, or by taking material from already discharging lesions. Specimens were usually sent quickly by road to the Central Public Health Laboratory at Wakefield. Altogether 21 specimens for bacteriological investigation were obtained with the results shown in Table 1.

The clinical appearance of the spreading cellulitis when in a severe form was suggestive of a streptococcal lesion, but the only organisms isolated from these and other cases were staphylococci. A high proportion of these (13 out of 17 ) were fully sensitive to penicillin. Where penicillin was unsuitable or inadvisable there was a choice of alternatives.

Treatment.-Except in the milder or resolving cases treatment included the intramuscular injection of procaine penicillin without waiting for the bacteriologist's report, cessation of work with rest and elevation of the affected limb, simple instruction in quadriceps exercises, and a period of intermediate work before the patient knelt again. When considered desirable antibiotic treatment was supplemented with a short course of oral sulphonamides.

Intramuscular procaine penicillin in oil, injected into the vastus lateralis, was given in 27 cases in the series. The first 15 received an injection of 600,000 units, increased in the last 12 cases to 900,000 units. When patients were unlikely to see their private doctors for a day or so, supportive treatment was given in the form of triple sulphonamides by mouth Thirteen patients were given such supportive treatment, each receiving $4 \mathrm{~g}$. per day for two days.

The importance of early and adequate treatment of infections about the knee still needs emphasis. Further work on the sensitivity to established and newer antibiotics of staphylococci prevailing in coal-faces with thin seams might provide the basis for a systematic outline for the treatment of staphylococcal lesions. Experience in the collection of the present cases suggests that there is need for such guidance. The use of long-acting penicillin, or of penicillin in combination with another antibiotic, would doubtless receive consideration.

Shift Loss.-The time off all duty for cases in the inflammatory group averaged 14 days (10 shifts). On discharge 30 men did no alternative work which did not entail kneeling, but resumed their normal occupations. Seven performed alternative work for an average period of seven days: four men lost no time at all and two more were excluded.

Many factors besides severity of the lesion treated have a bearing on the amount of working time lost. Advice not to resume kneeling work immediately on discharge after treatment was frequently unwelcome, as alternative or standing work seriously reduces a faceworker's earning capacity.

Occupation.-The occupations of the men in the inflammatory group were: 22 fillers, five rippers, four beltmen, four borers, two deputies, and six with various other occupations.

\section{Pressure Lesions}

A total of 51 pressure lesions with effusions into bursae was seen, with the following anatomical distribution:

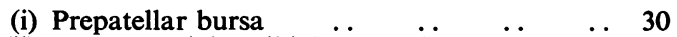

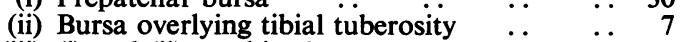

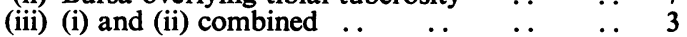

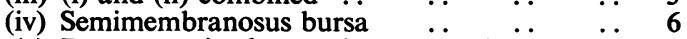

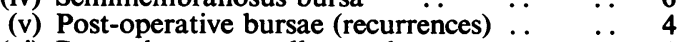

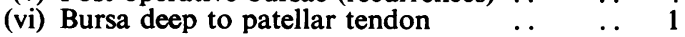


Although pressure is a form of trauma, a distinction was made between the insidious onset of lesions which appeared to be due to prolonged occupational pressure, and those due to ordinary trauma; the latter for purposes of this classification was restricted to a clearly remembered pain-eliciting incident. Pressure lesions made themselves known by the appearance of an effusion into a bursa. In most cases patients stated that during the course of a shift they became aware of increasing discomfort in one knee while kneeling. Examination of the knee disclosed a swelling. By this time disability was considerable and kneeling was impossible.

As expected the prepatellar bursa was affected most commonly, but the appearance of effusions into the bursa overlying the tibial tuberosity was also familiar, and when present made kneeling very painful. Semimembranosus bursae when enlarged interfered much less with kneeling, but caused anxiety by their presence and a sense of discomfort on full flexion.

Clinical findings were typically a cold effusion into a bursa related to an otherwise normal knee joint. In acute cases the walls of the enlarged bursa were thin, but thickened and inelastic walls can occur in chronic cases, sometimes with crepitus resembling that of tenosynovitis. A few acute effusions were accompanied by reaction with slight local heat similar to that seen in traumatic effusions in joints, but in these as in the "cold " effusions the cause was the same, namely an aseptic inflammation of the bursa.

The estimated contents of the bursae varied from 2 or $3 \mathrm{ml}$. to much larger quantities, the most usual estimates being between 10 and $30 \mathrm{ml}$. in the three bursae most commonly affected.

In four cases the enlargement of bursae was a late post-operative recurrence with representatives of prepatellar, pretibial, and semimembranosus bursae included. In only one case was a typically dumb-bell shaped swelling of the bursa deep to the patellar tendon seen.

Apart from effusions into bursae, some occupational "thickening" is commonly observed when the knees of miners working at thin seams are examined. A careful examination of the knees of 51 rescue men (43 who were asked estimated that $64 \%$ of their working time was spent kneeling) showed that only 20 had normal knees. In 29 there was a clinical impression of a small excess of prepatellar fluid, and in two there was diffuse subcutaneous thickening. Thirty-three men were examined on the coal-face (estimated kneeling time $73 \%$ of shift), and in only 12 of these were the knees normal. In 17 there appeared to be some excess of prepatellar fluid, and in four there was diffuse thickening.

These findings indicate that small amounts of fluid in one or more prepatellar bursae, and other pressure effects, are probably common in miners working at thin seams and are compatible with full efficiency.

In considering the knee which has failed under pressure, it may be accepted that it is the appearance, accompanied by pain, of a substantial effusion into a bursa which forces the patient to seek medical advice. Remembered trauma played a negligible part in this series. The effusion is a complication of prolonged weight transmission through joint surfaces not intended for such stresses. A situation has developed in which the knee has been tested beyond the limit of adaptation, and pressure intolerance is the outcome. This explanation would apply to effusion into the semimembranosus bursa, as well as those most commonly affected, but sheds no light on the haemorrhagic nature of the effusion, an unexpected fact established regularly by aspiration.

Persistent thickening in the region of the tibial tuberosity has been seen in a number of cases receiving treatment for the knees and is alluded to by Watkins and Hunt (1954). In some cases the prominence was sufficiently obvious to suggest the desirability of radiological confirmation. Radiographs of the knee joint were taken in seven cases and thickening of the tibial cortex showed in four of these. In four radiographs there appeared to be an exaggeration, marked in one case, of the normal backward inclination of the proximal extremity of the tibia, which itself would make the tuberosity more prominent, and suggests the possibility of a plastic alteration in shape as an effect of prolonged weight bearing. This appearance is not thought to be a result of former osteochondritis.

Treatment.-Treatment consisted of cessation of all kneeling work, aspirations of the swelling if large, followed by firm bandaging, complete rest, and elevation of the limb in the more severe cases, quadriceps exercises, and, when men could be persuaded to adopt it, a period of intermediate work before resumption of kneeling. Aspiration was performed through a 17- or 16-gauge needle inserted after procaine infiltration.

Altogether bursae were aspirated in 12 cases. In five cases aspiration had to be repeated, and in one patient the same bursa was aspirated seven times. The amounts recovered at one time from the prepatellar bursa varied from $6 \mathrm{ml}$. to $63 \mathrm{ml}$. Smaller amounts were withdrawn from the bursa over the tibial tuberosity, usually only a few millilitres, but as much as $30 \mathrm{ml}$. has been obtained from this bursa. 
The total number of aspirations and re-aspirations of bursae was 22, and in 17 instances in which the fluid was sent to the laboratory for bacteriological examination, no organisms were obtained on culture.

Aspiration fluid was remarkable for being visibly, usually heavily, bloodstained, in all the above cases but one; re-accumulated fluid continued to be haemorrhagic, even days after cessation of kneeling. Notwithstanding the almost constant finding of blood-stained fluid in these bursae, trauma was not an exciting cause. This finding may point to damage to the endothelial lining of the bursa as a late effect of pressure.

Aspiration appeared to be of value in treatment, and should be performed early in cases with sufficient fluid to justify it, and repeated if necessary. Aspiration should not be done, however, if the overlying skin is macerated or infected, nor in cases of effusion into the semimembranosus bursa, owing to its communication with the knee joint. The injection, after aspiration, of hyaluronidase may prove to be a useful addition to treatment, and the use in the same way of insoluble hydrocortisone may considerably hasten recovery. The intra-articular injection of hydrocortisone for sprains of the knee has been favourably reported upon by Cualacci and Bernadou (1956).

A period of work not requiring kneeling to permit full resolution of inflammatory, and especially pressure, lesions is an important step which is insufficiently appreciated and is often difficult to enforce. It is thought that many recurrences and conversions to chronic status of effusions into bursae arise from a premature return to work on the knees.

For refractory cases, especially in younger men, radical excision of the affected bursa may be the best course to advise. Thirteen examples of surgical treatment were seen in this survey. Six were successful with three men still kneeling at work 20, 10, and four years after excision of a prepatellar bursa; the other three cases were more recent but the patients had resumed kneeling two, six, and 15 months after operation. In one case operation was too recent for assessment; kneeling had been resumed four months after excision of a prepatellar bursa and was still causing pain two months later. The result of operation was disappointing in the remaining six men. Two still complained 22 years and 11 years after excision of a prepatellar bursa, and four stated that they were unable to work on their knees $27,20,15$, and eight months after operation. The last case was prevented from kneeling by the development of an aseptic periostitis in the region of the tibial tuberosity. A post-operative recurrence of prepatellar and pretibial fluid swelling has been noted in a few cases, and one semimembranosus recurrence has been seen. Operation requires a course of rehabilitation and freedom from kneeling which may be prolonged, and it is to some extent an open question whether the protracted rest from kneeling might not have been equally effective without operation. Only the most severe cases reach the surgeon, and in some it is likely that resort has only been had to surgery when the patient's knees had lost their last vestige of tolerance to pressure, and would soon have compelled a change of occupation with or without operation.

Shift Loss.-Omitting those who could not be followed up because they failed to keep under observation or left kneeling work permanently or left the mine, and also omitting post-operative cases, there are records of $33 \mathrm{men}$. Their average loss of all work is 21 days. Twenty-three men returned to full kneeling work on discharge, a number with a small excess of fluid persisting in the affected bursa, and 10 put in a temporary period of work not requiring kneeling which averaged 24 days.

Occupation.-The occupations of the men with pressure lesions were as follows: 20 fillers, 14 rippers, five machine men, four borers, three beltmen, two shotfirers, two repairers, and one under-manager.

\section{Other Conditions}

There were eight cases classified as "other conditions", in which the complaint was not inflammatory and not a manifestation of pressure in a bursa. Three of these were due to severe trauma causing the rapid appearance of swelling in a bursa (two in bursae overlying the tibial tuberosity and one in both the pretibial and prepatellar bursae). In two of these cases blood was aspirated. One other was a sole example of patellar tendinitis which resolved rapidly on rest and salicylates. The remaining four had tender aseptic periostitis of the tibial tuberosity, which was quite severe in one with new tibial bone laid down and calcification in the patellar tendon, and was a serious occupational disability in all cases. In another the periostitis had developed post-operatively and in the patient's own words " the disease was on the bone". Periostitis and tendinitis do not, strictly speaking, constitute beat knee as defined in the Industrial Injuries Act.

\section{Prevention}

As long as men subject their knees to grossly unphysiological stresses, these stresses will continue to manifest themselves by the appearance of beat knee, either in its infective subcutaneous form or more deeply as a pressure lesion. 
Properly designed knee pads, by cushioning weight and harmonizing irregularities in the surfaces knelt upon, must continue to be the main defence against pressure lesions. All the patients in this series wore knee pads habitually and, it may be assumed, were convinced of their value. There is, in fact, no other protection available to the almost constant kneeler. The quality of knee pads varies considerably, and certain of the heavier and better designed types are efficient. Whilst knee pads protect against pressure lesions, it is possible that under unfavourable environmental conditions they may contribute to the risk of staphylococcal lesions by excluding ventilation, macerating the skin, and acting as a trap for sharp particles of coal. It may be noted that infected abrasions were only seen in three cases in the present series, and that in only two of these could the lesion have been caused by coal particles inside the knee pads.

If the general outlook as far as the prevention of beat conditions of the knee is unpromising, particularly as regards pressure lesions, the prospects for early skilled treatment are encouraging. To be effective a recommended outline of treatment should be authoritatively codified, and publicized as necessary. These measures could be combined with a campaign amongst miners emphasizing the advantages of very early treatment in beat knee.

\section{Summary}

One hundred and two consecutive cases of beat knee are described. All occurred in coal-miners working thin seams in the North Eastern (Yorkshire) Division of the National Coal Board.

Cases were classified as inflammatory (43), pressure lesions (effusions into bursae) (51), and other conditions (eight).

Inflammatory lesions presented as folliculitis, cellulitis, or both. In 17 cases with positive cultures staphylococci were obtained, and in 13 of these the organism was sensitive to penicillin.

Pressure lesions manifested themselves by effusions into bursae, the distribution being: prepatellar 30 , bursa overlying tibial tuberosity seven, the preceding two combined three, semimembranosus six, and miscellaneous affections of bursa five.

The aetiology of beat knee is discussed with particular reference to pressure lesions.

In examining the effects of pressure on the knee, it is noted that completely "normal" knees were only observed in 32 out of 84 faceworkers on thin seams who had no complaint of disability. The larger effusions into bursae which, with pain, distinguished the disabling knee, are thought to be due to an aseptic inflammation of the bursa, and appear to be the result of pressure intolerance arising from prolonged weight-bearing through joint surfaces never intended for such stresses.

In both main types of beat knee essentials in treatment include its institution at the earliest possible moment, and the prevention of kneeling both at the beginning of treatment and for some time after its conclusion, i.e., on first return to work. A case can be made for the early injection of adequate amounts of intramuscular penicillin in the inflammatory forms of beat knee, and it is recommended that the commoner types of effusion into bursae should be aspirated early, and if necessary repeatedly. Despite the absence of any history of trauma, aspirated and re-aspirated bursa fluid in this series was regularly blood-stained. It is possible that improvements in treatment would result from the use of the new long-acting penicillin preparations in inflammatory cases, and the injection of hyaluronidase or hydrocortisone in pressure lesions.

Experience suggests that the preparation of standardized schemes of treatment, appropriate to inflammatory and pressure lesions, would be of value, particularly if combined with simple propaganda amongst miners emphasizing the importance of early treatment.

I wish to thank Dr. J. M. Rogan, Chief Medical Officer of the National Coal Board, for his interest and encouragement during the preparation of this paper, and for permission to publish it. I am indebted to Dr. T. D. Spencer, Divisional Medical Officer, for his assistance in planning and carrying out this survey, as well as to Dr. H. T. Findlay and Dr. L. A. Little for their valuable work on the bacteriological aspects of the investigation, and to Dr. W. Davidson for the radiographs. No acknowledgement would be complete without mentioning Mr. J. H. Atkinson, the Area General Manager, and other officials, as well as Miss P. Jubb and Mr. W. Booth and the many helpers and miners in Area 6 of the North Eastern Division, without whose enthusiasm and cooperation the work would not have been possible.

\section{REFERENCES}

Cualacci, P., and Bernadou, J. (1956). Rev. méd. minière, 9, Nos. $30-31$, p. 20.

Watkins, J. T., and Hunt, T. A. (1954). Med. Press, 232, 5. 